\title{
summary
}

\section{Telephone prompts improve questionnaire response rates}

Rikard-Bell G, Ward J. Maximising response rates to a survey of dentists: a randomised trial. Austral Dent J 2000; 45:46-48

Objective To compare the effectiveness of advance telephone and letter prompts in maximising response rates from a questionnaire to dentists.

\section{Design Randomised controlled trial with Australian dental} practitioners.

Intervention An advance telephone prompt using a standard script was compared with an advance letter. If the dentist was unavailable for the first call, one further attempt was made after which a message was left. Two reminder letters and a telephone reminder were made to nonresponders. Responses were assessed at 15 and 65 days and a thank-you letter sent to dentists who returned completed questionnaires.

\section{Outcome measures Response rate at day 15 and cumulative} response at day 65 .

Results The overall response rate was $83 \%$. The response from the telephoned dentists was significantly higher than in the letter group ( $89 \%$ compared with $78 \%$ ). Calculating numbers needed to treat (NNT; see Table 1) shows that you would need to contact 10 dentists by telephone to induce one additional dentist to respond, although you may need to speak to between five and 42 .

Table 1 Summary of response by dentists to letter and telephone call

\begin{tabular}{lcc}
\hline & Telephone prompt & Postal prompt \\
\hline $\mathrm{n}$ & 111 & 111 \\
$\mathrm{n}$ excluding ineligible practitioners & 90 & 89 \\
Responders at day 15 & 48 & 30 \\
Responders at day 65 & 80 & 69 \\
Using intention-to-treat analysis & & \\
CER & \multicolumn{2}{c}{$62.2 \%$} \\
EER & $72.1 \%$ \\
NNT & $10(95 \% \mathrm{Cl} 5-42)$ \\
\hline
\end{tabular}

Conclusions Significantly higher response rates can be achieved by making telephone contact in advance of a conventional questionnaire mail-out.

Address for reprints: Associate Professor Jeanette Ward, CSAHS Needs Assessment and Health Outcomes Unit, Locked Bag 8, Newtown, New South Wales 2042, Australia.

\section{Commentary}

Non-response is the most important source of non-sampling error in surveys. Consequently, the response to a sample survey and the magnitude of bias induced by non-response are issues that need to be considered when using data derived from surveys. Although the extent of bias induced by failure to collect data from some of the individuals sampled is the main issue to be taken into account, the response rate is usually taken to be an indication of the quality of a survey. This randomised controlled trial was conducted to evaluate the effect of an advance telephone call (intervention group) compared with an advance letter (control group) in increasing the response rate by dentists to a mailed questionnaire concerning the role of dentists in providing smoking-cessation advice to their patients. An 8-page questionnaire and explanatory letter were mailed out to both groups 3 days after these initial prompts. Two reminder letters, an additional questionnaire and a telephone call were made to non-responders in both groups. After 65 days, response rates in the interventions groups were significantly higher than in the control group (89\% versus 78\%). According to guidelines published in the British Dental Journal the former can be considered to be good and the latter borderline acceptable-good.

Although the study clearly demonstrated the effect of the intervention on the response rate to the mailed questionnaire, it is not clear that an increase of $11 \%$ in the response was effective in reducing bias in estimates derived from the survey. Non-response error is a function of the response rate and the magnitude of the differences between responders and non-responders. Although the potential for bias decreases as the response rate increases, low response rates do not necessarily compromise the results of a survey.
Consequently, it would have been useful to have some information on differences in the characteristics of responders and non-responders in each of the groups and estimates of the degree of bias introduced by residual non-response. As the authors note, response-enhancement strategies such as advance telephone calls can be costly and time-consuming but may not reduce bias to an appreciable degree. Moreover, although an increase in the response rate will reduce the standard errors of estimates, the increase has to be large in order to have a substantial effect the precision of estimates. Accordingly, the costs and benefits of response-enhancement strategies need to be carefully appraised before they are implemented.

$$
\begin{aligned}
& \text { David D Locker } \\
& \text { Faculty of Dentistry, University of } \\
& \text { Toronto, Ontario, Canada }
\end{aligned}
$$

